\title{
Discrete-Time Circular Walking Pattern for Biped Robots
}

\author{
Seungchul Lim* and Young Ik Son ${ }^{\dagger}$
}

\begin{abstract}
When biped robots make turns fast, they may fall due to the action of centrifugal force. This is why one needs to consider the Zero Moment Point (ZMP) equations with respect to cylindrical coordinate system. Those ZMP equations are, however, so coupled and highly nonlinear even for the simple inverted pendulum model that it is hard to find a closed-form solution. Therefore, in this paper, they have been converted through temporal discretization to difference equations that admit numerical solution by most on-board computers. Thus-obtained walking patterns have been characterized and applied to several cases of different speed for comparison purposes. In so doing, the steady patterns have been blended with a type of transitional patterns to change the walking speed in the beginning and/or mid course of walking. Finally, those combined patterns have been put to test on a multi-body robot model by ADAMS ${ }^{\circledR}$. Test results show that the robot could walk along a sample circular path as predicted at rapid speeds despite some modeling error, distributed mass and ground contact effects, validating efficacy of the suggested approach.
\end{abstract}

Keywords: Walking pattern, Centrifugal force, Cylindrical coordinate system, Euler method, Inverted pendulum, ZMP

\section{Introduction}

The research on humanoid robots has been diverging into various categories including the robot development and many related studies, such as dynamic walking [1-3], passive walking [4], adaptive motion planning [5], remote control of mobile robots [6], path planning for obstacle avoidance [7, 8], walking pattern generation [9], just to name a few. Among them, control algorithm of walking pattern plays a significant role [9] because, either in a general or an emergent situation, robots may have to modify its preplanned body motion somewhat and somehow for stability by adjusting leg joint's angles or even its next footsteps based on sensory feedback signals. Many approaches have been formulated for the control of bipedal locomotion. (Refer to [10] for a recent review.)

Since all paths including straight lines are in fact a combination of various circular arcs, biped robots need to follow a circular path of a finite radius more often than not. From this point of view, their ability to walk stably and precisely along such a curve is crucial in particular for dynamic gaits [11-13]. Regrettably, however, many biped robots of today make turns relying on the patterns found by an ad hoc approach including straight-walking patterns modified only with pelvic yaw motions activated. Such practices without due attention to kinematics and dynamics peculiar to circular-path walking will certainly lead to either inaccuracy or instability as the speed of turning

\footnotetext{
$\dagger$ Corresponding Author: Dept. of Electrical Engineering, Myongji University, Korea. (sonyi@mju.ac.kr)

* Dept. of Mechanical Engineering, Myongji University, Korea. (slim@mju.ac.kr)

Received: March 28, 2015; Accepted: February 3, 2016
}

\section{further increases.}

In this regard, noteworthy is an existing study [14] that developed a method to enable free and easy acceleration/ deceleration or direction change by adjusting the footlanding position and rotating the new local reference frame to be placed there while maintaining the orbital energy of the walking. According to the method, one can obtain suitable walking patterns for any circular path, keeping constant both the stride length and the rotation angle of the local frame. But the relationship between stepping speed and stability is hard to catch from it because dynamic effects arising from the body turn were not elucidated. Meanwhile, a real-time steering algorithm has been disclosed for humanoid robots [15]. Using it, robots can robustly chase any target position and orientation of the body without troubles such as leg-crossing, excess stride, or deadlock. Still, it also overlooked the latent rotational body effects.

In addition, another work [13] was recently made public, in which ZMP [16] equations were derived with respect to a cylindrical coordinate system to take into account the rotating body effects explicitly, and appropriate walking patterns for an example circular path were proposed in form of continuous time functions. The approach worked out fine. However, those walking patterns take relatively heavy computations to be prepared and nevertheless are not solutions of the ZMP equations after all, leaving room for some improvement.

Therefore, herein a new attempt is to be made to find exact steady walking patterns and see their characteristics. To this end, the ZMP equations aforementioned [13] are to be quoted and properly modified. Moreover, the transitional walking patterns [13] that can smoothly 
connect distinct gaits will be also applied. When both are made ready, several walking patterns of different speed will be generated and characterized for a typical biped robot following a sample path. Besides, through comparisons of those the relationship between stepping speed and stability is to be investigated. Finally, those patterns will be tested on the full, multi-body robot constructed and dynamically analyzed by ADAMS [17].

\section{Robot and ZMP Equations}

Portrayed in Fig. 1 are the typical biped robot under consideration and its coordinate systems in use. The robot consists of an upper body and two symmetric legs with six revolute joints each. In fact, three joints at the pelvis and two joints at the ankle are modeled to intersect orthogonally at a point (i.e., one point in the pelvis and another in the ankle). Those joints are arranged in the sequence of yaw, roll, pitch, pitch, pitch, and roll from top to bottom. More specifically, the robot is $478 \mathrm{~mm}$ tall and assumed made of aluminum, having a mass of $5.89 \mathrm{~kg}$ with about $82 \%$ of its weight in the upper body. Refer to Table 1 for more details. As for coordinate systems, $\{0\}$ is defined the Cartesian inertial frame established on the ground, $\{C\}$ the cylindrical frame with origin at $o^{\prime}$ off from point $O$ by distance $r_{m}$, and $\{1\}$ the rectangular body-fixed frame initially parallel with $\{0\}$.

For a robot to be stable in motion, its ZMP must stay inside the support region, but such a ZMP constraint itself may take a different form depending on situations $[1,16]$. This holds alike for circular walking as was shown in [13]. The corresponding ZMP equations turned out to be Eq. (1), the robot being modeled as an inverted pendulum with its torso assumed to stay at a constant height and upright without any pitch or roll motions throughout walking:

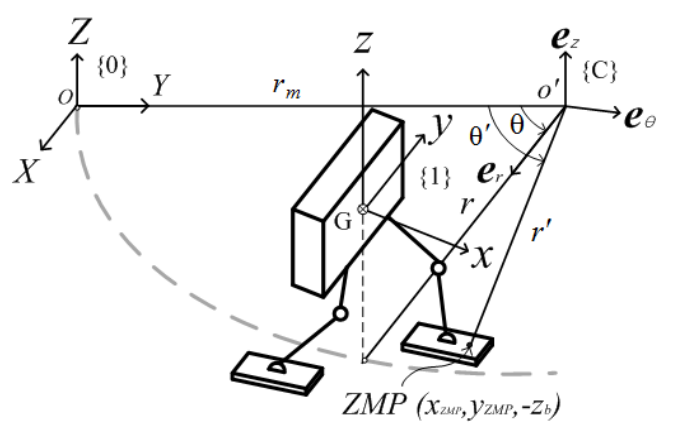

Fig. 1 Considered robot and its coordinate systems

Table 1. Specifications for the robot

\begin{tabular}{c|c|c}
\hline Part & Size $[\mathrm{mm}]$ & Mass[kg] \\
\hline Body & $96(\mathrm{~W}) \times 150(\mathrm{H}) \times 60(\mathrm{~T})$ & 4.833 \\
\hline Each Leg & $325(\mathrm{~L})$ & $4.860 \mathrm{e}-1$ \\
\hline Each Foot & $50(\mathrm{~W}) \times 110(\mathrm{~L}) \times 3(\mathrm{~T})$ & $4.197 \mathrm{e}-2$ \\
\hline Total & - & 5.889 \\
\hline
\end{tabular}

$$
\ddot{r}-r \dot{\theta}^{2}=a^{2} y_{Z M P}, \quad 2 \dot{r} \dot{\theta}+r \ddot{\theta}=-a^{2} x_{Z M P}
$$

in which $r$ and $\theta$ respectively represent the radial and angular body positions, $x_{Z M P}$ and $y_{Z M P}$ together the ZMP's horizontal coordinates measured in frame $\{1\}$, and $a\left(=\sqrt{g / z_{b}}\right)$ is a frequency-type parameter that remains constant with the gravitational acceleration, $g$, unless the body height, $z_{b}$, changes during locomotion. As is known, the inverted pendulum model acknowledges robot's mass only on the torso. Therefore it may lack sufficient reliability of outcomes from dynamic analyses of robots. Nevertheless, its kinds have been widely used for pattern synthesis or generation for good reasons $[1,9,14,19]$.

By nature $x_{Z M P}$ and $y_{Z M P}$, which are the input terms of Eq. (1), make hard the solution process since they are referenced by frame $\{1\}$ whose origin persists to move. Therefore, in this paper Eqs. (2) and (3) are successively employed to transform Eq. (1) to (4) which is totally expressed in terms of frame $\{C\}$.

$$
\begin{gathered}
\left\{\begin{array}{c}
x_{Z M P} \\
y_{Z M P}
\end{array}\right\}=\left[\begin{array}{cc}
c \theta & s \theta \\
-s \theta & c \theta
\end{array}\right]\left\{\begin{array}{c}
X_{Z M P}-r s \theta \\
Y_{Z M P}-r_{m}+r c \theta
\end{array}\right\} \\
X_{Z M P}=r^{\prime} s \theta^{\prime}, Y_{Z M P}=r_{m}-r^{\prime} c \theta^{\prime} \\
\ddot{r}-r\left(\dot{\theta}^{2}+a^{2}\right)=-a^{2} r^{\prime} c\left(\theta^{\prime}-\theta\right) \\
2 \dot{r} \dot{\theta}+r \ddot{\theta}=-a^{2} r^{\prime} s\left(\theta^{\prime}-\theta\right)
\end{gathered}
$$

In the equations above, $s$ and $c$ are the abbreviations for sine and cosine functions respectively, and $r^{\prime}$ and $\theta^{\prime}$ represent the ZMP's horizontal coordinates in frame $\{\mathrm{C}\}$.

\section{Walking Pattern Generation}

Not to mention Eq. (1), even the modified ZMP Eq. (4) is still a pair of differential equations not just coupled but also highly nonlinear as opposed to its counterpart in straight walking cases. So, it is not feasible to obtain closed-form solutions for $r$ and $\theta$ given ZMP inputs of $r^{\prime}$ and $\theta^{\prime}$.

Therefore, following the Euler method [18] let us discretize Eq. (4) in time. If the sampling period and arbitrary sampling instant are denoted by $T$ and subscript $k(=0,1,2, \ldots)$ respectively, the ZMP equations of concern will end up like Eq. (5), which is usable to find body motion during SSP (Single Support Phase).

$$
\begin{aligned}
r_{k+2}= & 2 r_{k+1}+\left\{\left(\theta_{k+1}-\theta_{k}\right)^{2}+(a T)^{2}-1\right\} r_{k} \\
& -(a T)^{2} r_{k}^{\prime} c\left(\theta_{k}^{\prime}-\theta_{k}\right) \\
\theta_{k+2}= & \frac{1}{r_{k}}\left\{-2\left(r_{k+1}-2 r_{k}\right) \theta_{k+1}+\left(2 r_{k+1}-3 r_{k}\right) \theta_{k}\right. \\
& \left.-(a T)^{2} r_{k}^{\prime} s\left(\theta_{k}^{\prime}-\theta_{k}\right)\right\}
\end{aligned}
$$

With initial conditions $\left(r_{0}, \theta_{0}, \dot{r}_{0}\right.$, and $\left.\dot{\theta}_{0}\right)$ and ZMP 
inputs $\left(r_{k}^{\prime}\right.$ and $\left.\theta_{k}^{\prime}\right)$ made available, the foregoing difference Eq. (5) can compute exact body motion by simple iterations, lending itself to real-time pattern generation. Undoubtedly, real-time pattern generation is preferable in that robots need not memorize vast walking pattern data designed offline and also they can be more adaptive to variable surroundings.

On the other hand, body motion during DSP (Double Support Phase) can be prescribed to be a uniform motion with continuous displacement and velocity across the boundary of neighboring SSPs. Then, with $v_{0}$ and $\omega_{0}$ defined as the radial and angular velocities at the start of DSP, it can be described as below.

$$
r_{k+1}=r_{k}+v_{0} T, \theta_{k+1}=\theta_{k}+\omega_{0} T
$$

Therefore, from Eq. (4) the corresponding ZMP position can be also acquired as in (7). At this point, it is noteworthy that the ZMP position concerned differs from the associated body position even during DSP because of the centrifugal force arising from body rotation. Yet, their difference may not be significant in many cases where $a>>\omega_{0}$ and $r_{k} a^{2}>2 v_{0} \omega_{0}$.

$$
\begin{gathered}
r_{k}^{\prime}=\sqrt{r_{k}^{2}\left(a^{2}+\omega_{0}^{2}\right)^{2}+\left(2 v_{0} \omega_{0}\right)^{2}} / a^{2} \\
\theta_{k}^{\prime}=\theta_{k}-\tan ^{-1} \frac{2 v_{0} \omega_{0}}{r_{k}\left(a^{2}+\omega_{0}{ }^{2}\right)}
\end{gathered}
$$

When time durations of SSP and DSP are denoted by $T_{S}$ and $T_{D}$ respectively, it is reasonable to determine $T_{S}$ as the time lapse of an interval over which the radial velocity becomes the negative of its initial value $v_{0}$, whereas $T_{D}$ as $2 \Delta r /\left|v_{0}\right|$, i.e., the ratio of radially traveled distance and speed during DSP.

By the way, to ensure the walking pattern continuous in terms of velocity and periodic as well during all SSPs in angular direction, both the initial and final angular velocities of each SSP have to be the same as $\omega_{0}$. However, this condition cannot be met necessarily by the solution of Eq. (5) with arbitrary ZMP inputs of $r_{k}^{\prime}$ and $\theta_{k}^{\prime}$. Therefore, instead of solving the equation for $\theta_{k}$, one might as well designate its rate as $\omega_{0}$ constantly, and set either $r_{k}^{\prime}$ or $\theta_{k}^{\prime}$ to a constant at the same time. Of the two choices, the former is more advantageous minimizing the possibility that robots fall down due to centrifugal force. Accordingly, in that case it is Eq. (5a) and Eq. (8a) derivable from $(4 b)$ or $(5 b)$ that govern the behavior of $r$ and $\theta^{\prime}$ during SSP now that $r^{\prime}$ and $\theta$ are merely inputs.

$$
\theta_{k}^{\prime}=\theta_{k}-s^{-1}\left(\frac{2 v_{k} \omega_{0}}{a^{2} r^{\prime}}\right)
$$

The variables, $\theta_{k}$ and $v_{k}$, in the equation above are readily obtained as by-products:

$$
\begin{gathered}
\theta_{k}=\theta_{0}+\omega_{0} k T \\
v_{k}=\left(r_{k+1}-r_{k}\right) / T
\end{gathered}
$$

During any steady turning, robots will have a periodic motion. However, since they tend to change gaits from one to another, some other walking pattern is also necessary for smooth transitions. So, a cubic polynomial in Eq. (9) is adopted not only for the transition between the initial stationary stance and the steady gait as before [13] but also between steady gaits of different speed. Its coefficients are determined by the position and speed at the initial (when $\tau=0$ ) and terminal (when $\tau=T_{t}$ ) moments of each transition, both of which are desired to belong to a DSP for larger stability margin.

$$
\begin{gathered}
r(\tau)=c_{3} \tau^{3}+c_{2} \tau^{2}+c_{1} \tau+c_{0} \\
\theta(\tau)=d_{3} \tau^{3}+d_{2} \tau^{2}+d_{1} \tau+d_{0}
\end{gathered}
$$

Similarly to Eq. (7), the ZMP position during such a transitional period can be identified as follows from Eq. (4).

$$
\begin{gathered}
r^{\prime}=\sqrt{\left(r a^{2}-\ddot{r}+r \dot{\theta}^{2}\right)^{2}+(2 \dot{r} \dot{\theta}+r \ddot{\theta})^{2}} / a^{2} \\
\theta^{\prime}=\theta+\tan ^{-1} \frac{2 \dot{r} \dot{\theta}+r \ddot{\theta}}{\ddot{r}-r\left(a^{2}+\dot{\theta}^{2}\right)}
\end{gathered}
$$

\section{Stability Criteria}

Illustrated in Fig. 2 is the logical flow that briefly sums up the aforementioned details of pattern generation in a sequential and iterative fashion and that can be used for automatic pattern generation. Prior to the start of actual walking cycle stability of initial patterns, transitional and steady, should be thoroughly checked. If any stability problem occurs, they need be modified by adjustment of input variables at the stages marked with shade. Once the stability issue is well resolved, only the other stages will run for robots to keep steady walking.

As for the stability criterion concerned, there are three kinds. The first, associated with the transitional walking pattern, is

$$
\left|\theta^{\prime}\right|<\min (\gamma, \delta)
$$

where $\gamma$ and $\delta$ as sketched in Fig. 3 indicate the angles bounding the range for $\theta^{\prime}$ to stay for the sake of stability. Meanwhile, since $r^{\prime}$ usually remains between two feet, the stability check in the radial direction can be omitted.

The second kind of stability criterion, which is associated with SSP, is one of Eq. (12), depending on which foot gives support. 


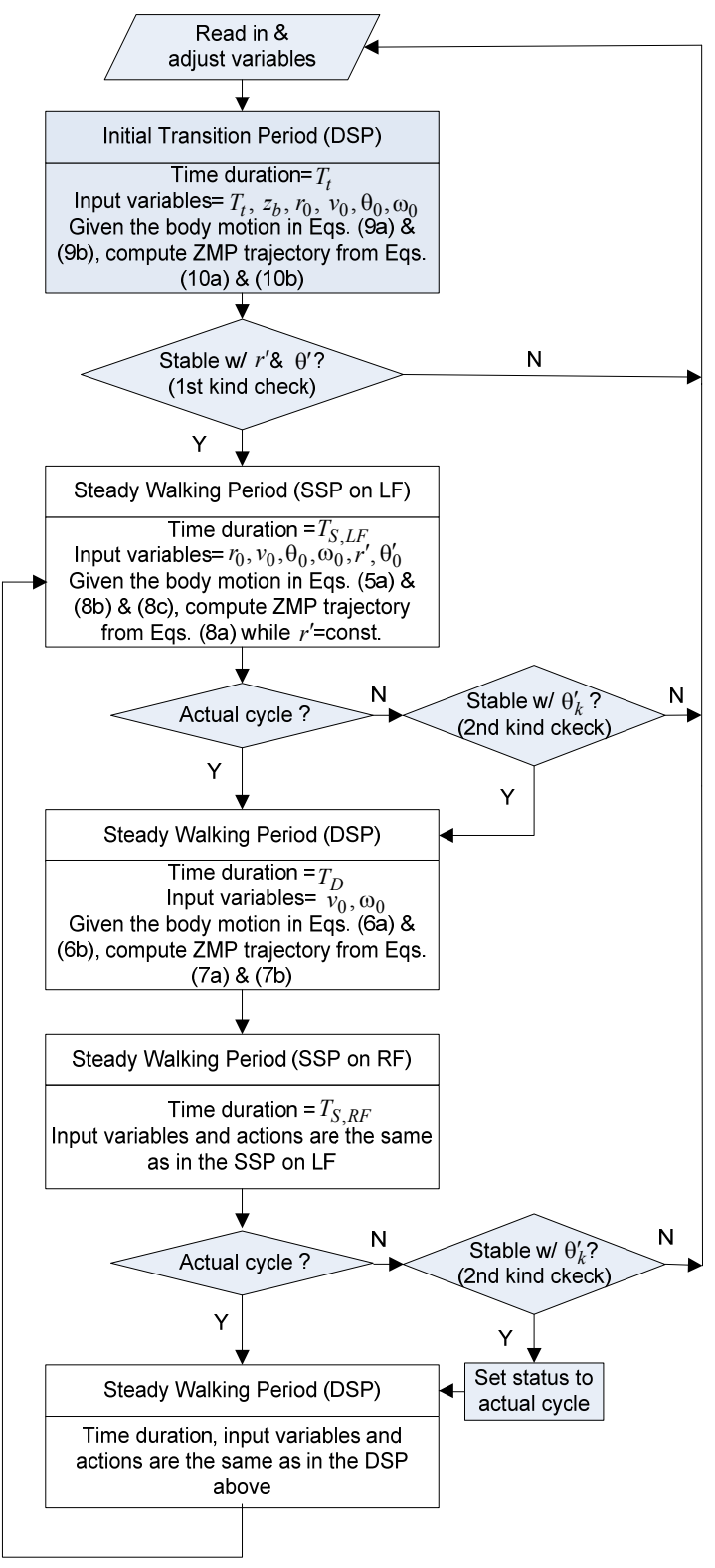

Fig. 2 Flow chart of walking pattern generation

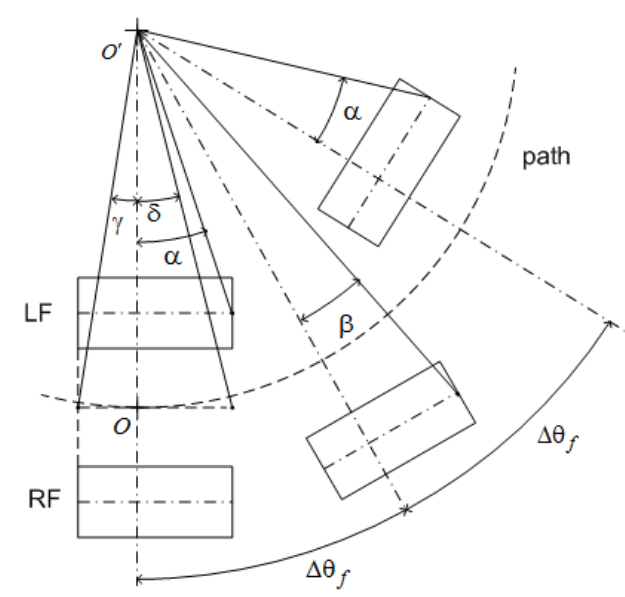

Fig. 3 Footprints and related angles

$$
\omega_{0} T_{S, L F}<\alpha \text { or } \omega_{0} T_{S, R F}<\beta
$$

where $\alpha$ and $\beta$ are the angles specified in Fig. 3 together with $\Delta \theta_{f}$, the uniform step angle. The good reason why Eq. (12) can be used is that $\Delta \theta^{\prime} \cong \Delta \theta=\omega_{0} T_{S}$. During DSP, stability need not be checked in common cases as mentioned earlier. The two stability criteria discussed hereto have much to do with foot size. However, the third one comes from another fact that there could be an upper limit to the rotating speed regardless of foot size. This will be treated in the next section.

\section{Dynamic Simulations}

In here, three walking patterns of different speed are generated at first by Eqs. (5a) and (6) (10), and then their characteristics including stability are comparatively examined. In addition, created also are two more patterns of composite speed that begin with relatively low speed to secure stability but later on maintain a raised speed. Then, all those patterns which are basically from the inverted pendulum model are put to test on the multi-body robot by ADAMS in the presence of modeling error, distributed mass and ground contact effects. In the process, an existing balance control [13] too is applied particularly during the initial transition period to prevent the robot from tipping.

\subsection{Inverted pendulum model}

The walking conditions are designated such that $z_{b}=350 \mathrm{~mm}, r_{m}=333 \mathrm{~mm}, \Delta r=25.56 \mathrm{~mm}, \Delta r^{\prime}=68$ $\mathrm{mm}, \theta_{0}=0, \Delta \theta_{f}=\pi / 15, \omega_{0}=0.1 \pi \mathrm{rad} / \mathrm{s}, T=10^{-4} \mathrm{~s}$, $T_{t}=1 \mathrm{~s} \quad$ with $\quad r_{0}=r_{m}+(-1)^{i+1} \Delta r, \quad r^{\prime}=r_{m}+(-1)^{i+1} \Delta r^{\prime}$, $v_{0}=(-1)^{i+1} 130.88 \mathrm{~mm} / \mathrm{s}$, and $\theta_{0}^{\prime}=i \Delta \theta_{f}$ for the $i$-th step when the right foot is the first step to take. Thus-obtained values of $T_{S}$ are listed in the first row of Table 2, showing that time duration of the left-foot (LF) support phase is slightly shorter than that of right-foot (RF) support phase. In contrast, $T_{D}$ turned out identically $0.3908 \mathrm{~s}$ so that the period of steady walking of $1 \mathrm{x}$ speed is $1.2871 \mathrm{~s}$.

Such discrepancy between the LF and RF supported SSP time durations may aggravate the stability in that the right foot has to move faster than the left despite its longer stride resulting from the sample path curved leftward. Moreover, it tends to get bigger with the speed. See Table 2. This phenomenon can be explained with Eq. (4a). When $\ddot{r}_{L F}$ and $\ddot{r}_{R F}$ denote the radial accelerations during LF and RF supported SSPs respectively, since $r \cong r_{m}+(-1)^{i+1} \Delta r$,

Table 2 Time durations of designed walking patterns

\begin{tabular}{c|c|c|c}
\hline Case & $T_{S}$ on LF $[\mathrm{s}]$ & $T_{S}$ on RF [s] & $T_{D}[\mathrm{~s}]$ \\
\hline 1x speed & 0.2432 & 0.2623 & 0.3908 \\
\hline 1.5x speed & 0.1590 & 0.1793 & 0.2606 \\
\hline 2x speed & 0.1097 & 0.1318 & 0.1955 \\
\hline
\end{tabular}


$\dot{\theta}=\omega_{0}, \theta^{\prime} \cong \theta$, and $r^{\prime}=r_{m}+(-1)^{i+1} \Delta r^{\prime}$, that equation can be approximated as below in the first place.

$$
\begin{aligned}
& \ddot{r}_{L F} \cong\left(r_{m}-\Delta r\right)\left(\omega_{0}^{2}+a^{2}\right)-a^{2}\left(r_{m}-\Delta r^{\prime}\right) \\
& \ddot{r}_{R F} \cong\left(r_{m}+\Delta r\right)\left(\omega_{0}^{2}+a^{2}\right)-a^{2}\left(r_{m}+\Delta r^{\prime}\right)
\end{aligned}
$$

Therefore, if the walking speed is low enough such that $\omega^{2}<<a^{2}$, it will hold that $\ddot{r}_{L F}=-\ddot{r}_{R F}=a^{2}\left(\Delta r^{\prime}-\Delta r\right)$. Otherwise, however, the magnitude difference between those accelerations becomes $2 r_{m} \omega_{0}{ }^{2}$, accounting for the consistent tendency.

On the other hand, if Eq. (13) is rearranged as follows, the third stability criterion can be better revealed.

$$
\begin{aligned}
& \ddot{r}_{L F} \cong\left(r_{m}-\Delta r\right) \omega_{0}^{2}+a^{2}\left(\Delta r^{\prime}-\Delta r\right) \\
& \ddot{r}_{R F} \cong\left(r_{m}+\Delta r\right) \omega_{0}{ }^{2}+a^{2}\left(\Delta r-\Delta r^{\prime}\right)
\end{aligned}
$$

Since $r_{m} \geq \Delta r$ and $\Delta r^{\prime}>\Delta r$ in general, $\ddot{r}_{L F}$ may be regarded always positive, but that is not the case for $\ddot{r}_{R F}$. As the rotating speed $\omega_{0}$ increases, it will change sign from negative to positive as graphed in Fig. 4 for the present case. The speed at which $\ddot{r}_{R F}$ becomes null is termed a critical value of $\omega_{0}$ and can be found as in Eq. (15). So, above it the circular walking will be unstable due to the lack of centripetal recovering motion.

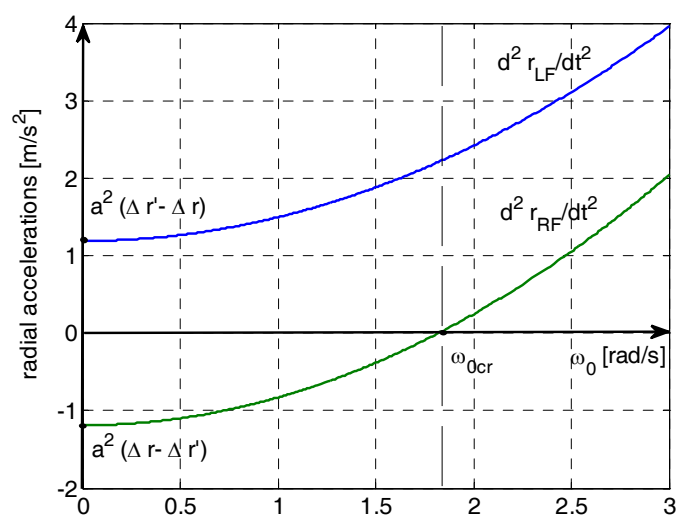

Fig. 4 Radial accelerations vs. rotating speed

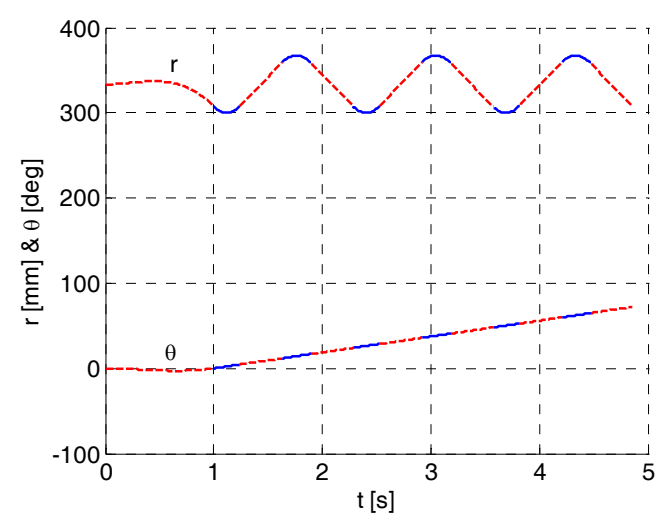

Fig. 5 Radial \& angular body positions

$$
\omega_{0 c r}=\sqrt{\frac{a^{2}\left(\Delta r^{\prime}-\Delta r\right)}{r_{m}+\Delta r}}
$$

During the entire simulation time of 4.86 s including the 1s long initial transition period, the robot advanced 6 steps. The corresponding time histories of body positions and body speeds are shown in Figs. 5 and 6, whereas those of body positions observed in frame $\{0\}$ and ZMP are on display in Figs. 7 and 8 respectively. Note that the dotted portion of each graph in Figs. 5 8, 10 and 11 to appear stands for trajectories during DSP.

Meanwhile, the foot motion is smoothly planned in frame $\{C\}$ according to the timing above and inputted $r^{\prime}$

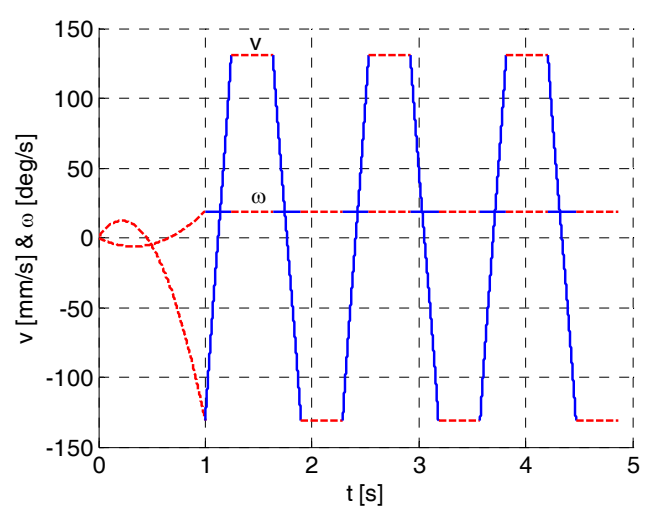

Fig. 6 Radial \& angular body speeds

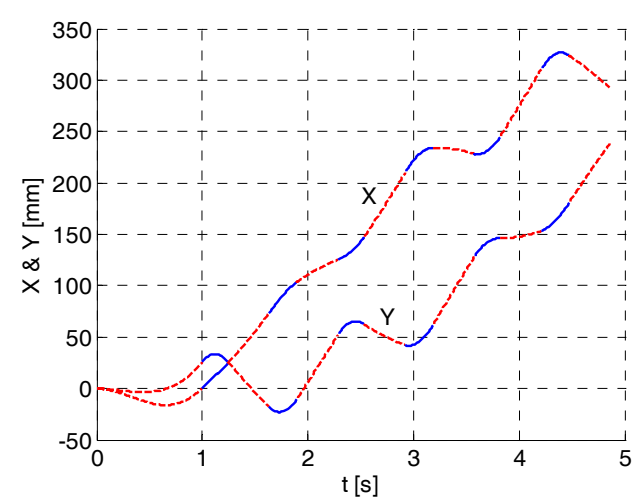

Fig. 7 Body positions in frame $\{0\}$

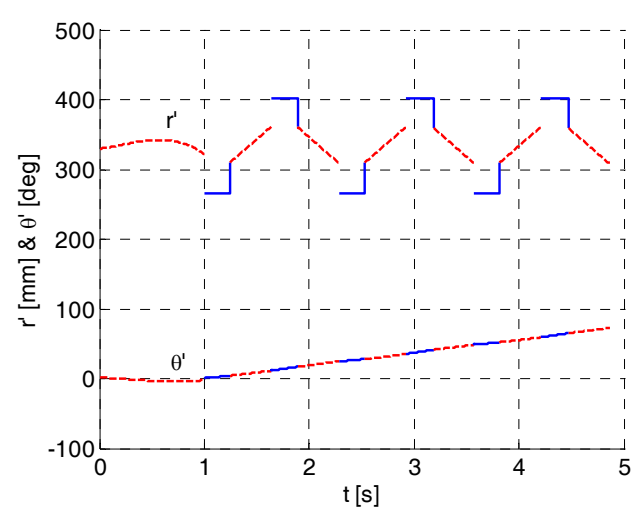

Fig. 8 ZMP trajectories 
and $\Delta \theta_{f}$. The result of walking based on such a pattern is shown at the top plot of Fig. 9, confirming that throughout the walking period ZMP continues to stay in the stable region, and the body proceeds along the circular path with a cyclic side movement during steady walking. In the figure the middle plot corresponds to the $1.5 \mathrm{x}$ nominal speed case implemented with $1.5 \omega_{0}, 0.6 \Delta r$, and $0.9 v_{0}$, whereas the bottom the double speed case with $2 \omega_{0}$, $0.4 \Delta r$, and $0.8 v_{0}$. Those two walking patterns of higher speed feature the unchanged stride length and proportionally reduced side motion.
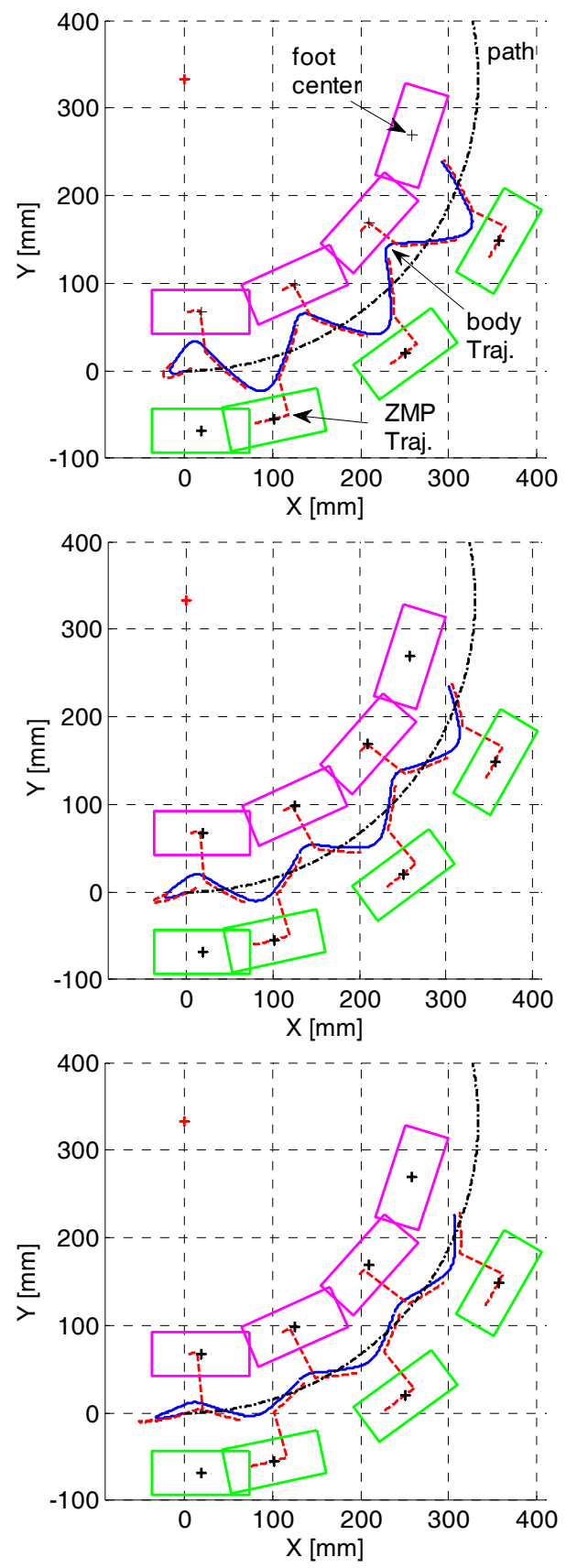

Fig. 9 Body \& ZMP trajectories in three speed cases: (top) $1 \mathrm{x}$, (middle) $1.5 \mathrm{x}$, (bottom) $2 \mathrm{x}$
In Fig. 9, it is apparent that steady walking is stable regardless of speed, but an instability problem occurs during the initial transient period except for the $1 \mathrm{x}$ speed case. In addition, comparison of ZMP trajectories during SSP of the three cases tells that they elongate in the tangential direction but do not change in the radial. This is the targeted robustness against the possibility that ZMP may shift centrifugally decreasing stability margin with speed raise. Owing to the property, even the steady walking pattern of $4 x$ speed has ever been checked stable although the margin was narrow.

In fact, Fig. 9 plays the role of visual tests of the two stability criteria in Eqs. (11) and (12), whereas the third one, Eq. (15), gives $\omega_{0 c r}$ of $1.87 \mathrm{rad} / \mathrm{s}$ which amounts to $5.9 \mathrm{x}$ speed in the present case.

In order to avoid the instability during the initial period, it seems more natural for robots to start walking at a relatively low speed and then speed up gradually in the course. From this perspective, an attempt is made that our robot takes the first step at the nominal speed but switches to $1.5 \mathrm{x}$ or $2 \mathrm{x}$ speed during the very next DSP, using the transitional pattern in Eq. (9) once again. As a result of the scheme the body moved as shown in Fig. 10, and the corresponding ZMP trajectories stayed inside the stable region throughout the trip as evidenced by Fig. 11 .

\subsection{Multi-body robot model}

Two subsequent figures relate to the simulation results by ADAMS; Fig. 12 is a collection of snap shots taken
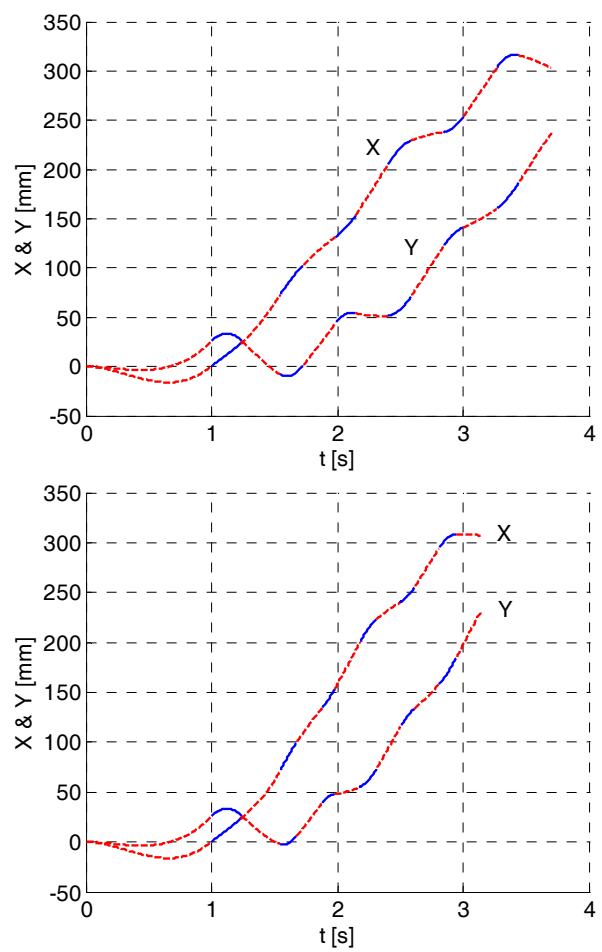

Fig. 10 Body positions in two composite speed cases: (top) $1 \& 1.5 \mathrm{x}$, (bottom) $1 \& 2 \mathrm{x}$ 

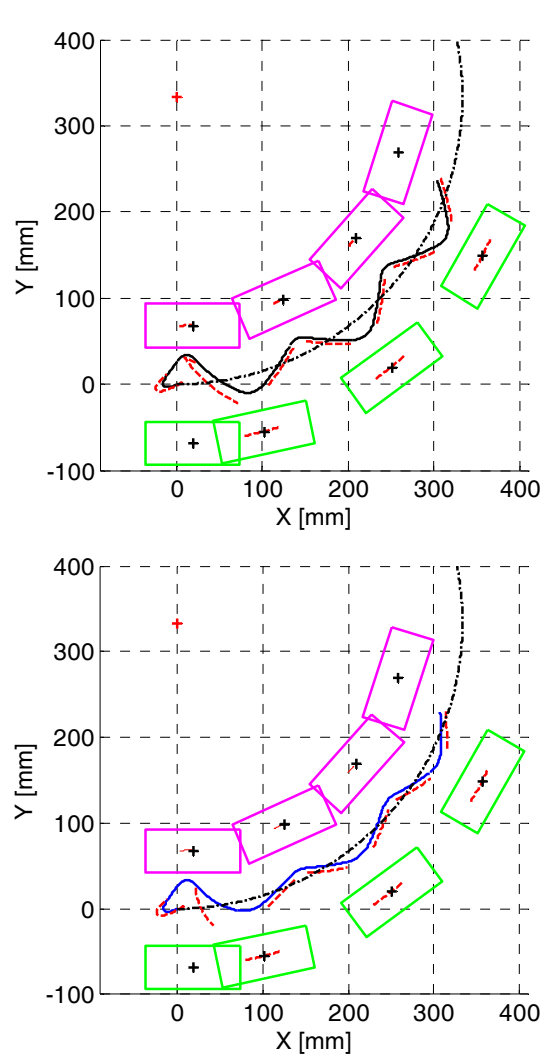

Fig. 11. Body \& ZMP trajectories in two composite speed cases: (top) $1 \& 1.5 \mathrm{x}$, (bottom) $1 \& 2 \mathrm{x}$

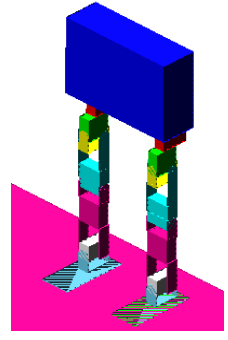

0s

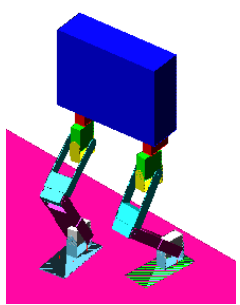

$2.5 \mathrm{~s}$

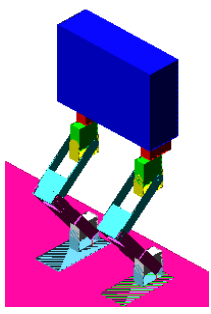

$1.0 \mathrm{~s}$

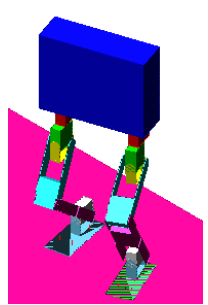

$3.0 \mathrm{~s}$

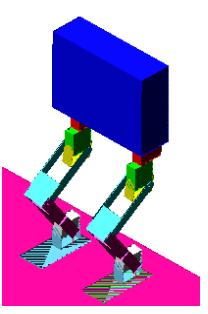

$2.0 \mathrm{~s}$

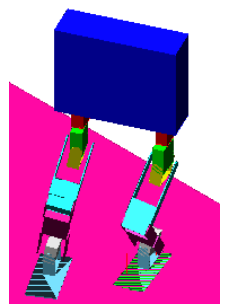

$3.5 \mathrm{~s}$
Fig. 12. Snapshots of the robot in circular walking

from an animated motion that lasted for about $6 \mathrm{~s}$ including the initial 1s long squatting followed by 1s long gait transition. It shows that the robot could walk at a speed of $0.4 \mathrm{~km} / \mathrm{h}$ and turn $12^{\circ}$ per step during steady walking. Although performance of the balance control applied during the initial $2 \mathrm{~s}$ is not presented here for brevity, it will
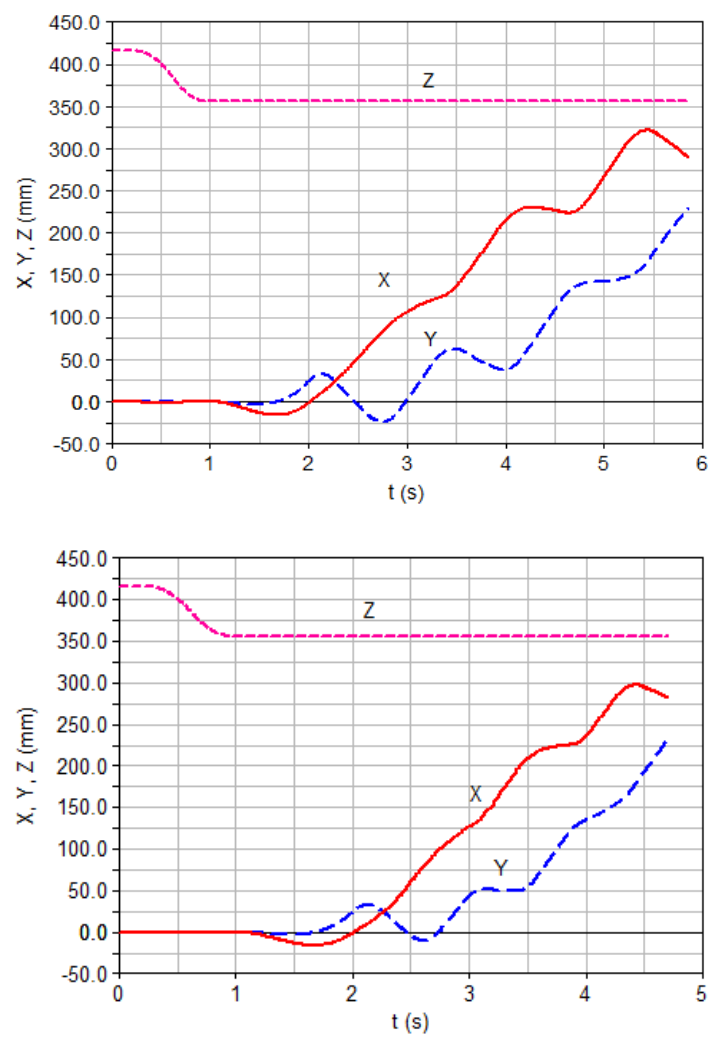

Fig. 13. Body positions from ADAMS simulation: (top) $1 \mathrm{x}$ speed case, (bottom) composite $1 \& 1.5 \mathrm{x}$ speed case

be informative to note that $1.5 \mathrm{x}$ and $2 \mathrm{x}$ speed cases as well as the nominal speed case also succeeded in entering the steady walking period owing to it in spite of some modeling error [13].

Fig. 13 carries two plots of the body position trajectories observed in frame $\{0\}$ : one in the nominal speed and the other in the composite $1 \& 1.5 \mathrm{x}$ speed case. Comparing data of Fig. 13 after the initial 1s in which the robot squats down with Figs. 7 and 10's upper plot, one can state that the gaits have been performed as planned with almost null and negligible errors respectively in the nominal and composite $1 \& 1.5 x$ speed cases. However, in the case of composite $1 \& 2 \mathrm{x}$ speed, the walking went unstable since the third step contrary to the prediction from Figs. 10 and 11. It happened because the right leg had to swing fast to complete its motion within even shorter time at that moment, giving rise to greater centrifugal force than preceding steps.

\section{Conclusions}

We have found an exact dynamic walking pattern necessary for biped robots to walk stably and precisely along circular paths. To this end, the ZMP equations have been fully derived with respect to a cylindrical coordinate 
system with its origin at the path's center and solved through temporal discretization. In addition, characteristics of the resultant walking patterns were analyzed phase by phase, and three stability criteria were formulated essential for automation of the iterative pattern generation process.

Finally, along with a simple and versatile transient walking pattern and an existing balance control, the designed walking patterns were tested on a typical robot by ADAMS in the presence of modeling errors, distributed mass, and ground contact effects. As a result, it was discovered that the robot of height $478 \mathrm{~mm}$ could successfully walk along a circular path of radius $333 \mathrm{~mm}$ at a speed up to $1.5 \mathrm{x} \sim 2 \mathrm{x}$ speed or $0.6 \sim 0.8 \mathrm{~km} / \mathrm{h}$.

Based on these we conclude that when combined, the suggested ZMP equations, the associated pattern generation process, and the resultant walking patterns can be very effective means for biped robots to perform stable and precise circular walking of high speed.

\section{Acknowledgements}

This research was supported by Basic Science Research Program through the National Research Foundation of Korea(NRF) funded by the Ministry of Education (NRF2013R1A1A2062370).

\section{References}

[1] S. Lim, "Dynamic Stair Walking of Biped Humanoid Robots," Journal of Mechanical Science and Technology, vol. 21, pp. 970-975, 2007.

[2] S. Lim, S.N. Oh and K.I. Kim, "Balance Control for Biped Walking Robots Using Only Zero-MomentPoint Position Signal," Electronics Letters, vol. 48, no. 1, pp. 19-20, Jan. 2012.

[3] D. J. Braun, J. E. Mitchell, and M. Goldfarb, "Actuated Dynamic Walking in a Seven-Link Biped Robot," IEEE/ASME Trans. Mechatronics, vol. 17, no. 1, pp. 147-156, Feb. 2012.

[4] M. R. Sabaapour, M. R. Hairi Yazdi, and B. Beigzadeh, "Toward Passive Turning in Biped Walkers," Procedia Technology, vol. 12, pp. 98-104, 2014.

[5] Z. Lu, F. Silva, Y. Zhang, Q. Wang, and L. S. Lopes, "A COP-based Controller for Adaptive Motion Planning of Single-Legged Robot," Proc. Int. Conference on Robotics and Biomimetics, pp. 42-47, Dec. 2012.

[6] D. Q. Khanh and Y. Suh, "Mobile Robot Destination Generation by Tracking a Remote Controller Using a Vision-aided Inertial Navigation Algorithm," Journal of Electrical Engineering \& Technology, vol. 8, no. 3, pp. 613-620, 2013.
[7] T. Kang, M. Lim, G. Park, and D. Kim, “3D VisionBased Local Path Planning System of a Humanoid Robot for Obstacle Avoidance," Journal of Electrical Engineering \& Technology, vol. 8, no. 4, pp. 879-888, 2013.

[8] S. Chang and U. Huh, " $G^{2}$ Continuity Smooth Path Planning using Cubic Polynomial Interpolation with Membership Function," Journal of Electrical Engineering \& Technology, vol. 10, no. 2. pp. 676-687, 2015.

[9] B. Lee and K. Kim, "Modifiable Walking Pattern Generation Handling Infeasible Navigational Commands for Humanoid Robots," Journal of Electrical Engineering \& Technology, vol. 9, no. 1, pp. 334-351, 2014.

[10] Y. Hurmuzlu, F. Genot, and B. Brogliato, "Modeling, Stability and Control of Biped Robots-A General Framework," Automatica, vol. 40, no. 10, pp. 16471664, 2004.

[11] S. Kohannim, and T. Iwasaki, "Optimal Turning Gait for Undulatory Locomotion," Proc. American Control Conference, Canada, pp.3459-3464, Jun. 2012.

[12] Y. J. Mon and C. M. Lin, "Supervisory Fussy Gaussian Neural Network Design for Mobile Robot Path Control," International Journal of Fuzzy Systems, vol. 15, no. 2, pp. 142-148, Jun. 2013.

[13] S. Lim, B. Kwak, J. Lim, and Y. Son, "Precision Circular-Path Walking of a Biped Robot with Consideration of Rotational Effects," Trans. the KSNVE, vol.24, no.4, pp.299-309, 2014.

[14] S. Kajita, F. Kanehiro, K. Kaneko, K. Fujiwara, K. Yokoi, and H. Hirukawa, "Biped Walking Pattern Generation by a Simple Three-Dimensional Inverted Pendulum Model," Advanced Robotics, vol. 17, no. 2, pp. 131-147, 2003.

[15] H. Kobayashi and T. Sugihara, "Self-consistent Automatic Navigation of COM and Feet for Realtime Humanoid Robot Steering," Proc. IEEE/RSJ Int. Conference on Intelligent Robots and Systems, pp. 3525-3530, 2009.

[16] M. Vukobratovic, B. Borovac, D. Surla, and D. Stokic, Scientific Fundamentals of Robotics 7: Biped Locomotion, Springer-Verlag, 1990.

[17] Basic ADAMS Full Simulation Training Guide, Mechanical Dynamics Inc, 2001.

[18] W. Press, S. Teukolsky, W. Vetterling, and B. Flannery, Numerical Recipes in Fortran, Cambridge University, 1992.

[19] Y. Hong, B. Lee, and J. Kim, “Command State-Based Modifiable Walking Pattern Generation on an Inclined Plane in Pitch and Roll Directions for Humanoid Robots," IEEE/ASME Trans. Mechatronics, vol.16, no.4, pp. 783-789, 2011. 


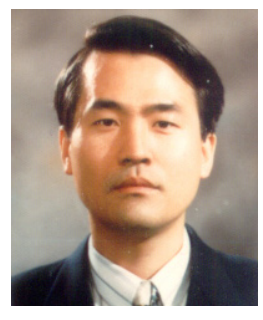

Seungchul Lim He received the B.S., M.S., and Ph.D. degrees from Seoul National University, KAIST, and Virginia Tech in 1981, 1983 and 1992 respectively. He is currently with ME department of Myongji University as a professor. His research interests include robotics and design of highperformance dynamic systems.

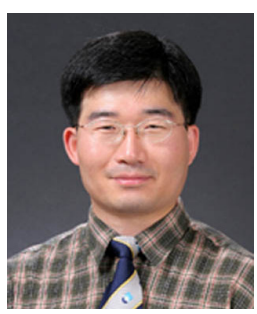

Young Ik Son He received the B.S., M.S., and Ph.D. degrees from Seoul National University, Korea, in 1995, 1997 and 2002, respectively. Since 2003, he has been with the Department of Electrical Engineering at Myongji University, Korea, where he is currently a professor. His research interests include robust controller design and its application to industrial electronics. 